\title{
Factores Asociados al Fracaso Escolar en la Educación Secundaria de Huelva
}

\author{
Associated Factors the School Failure in the Secondary \\ Education of Huelva
}

\author{
Carmen Márquez Vázquez * \\ Universidad de Huelva
}

\begin{abstract}
En este trabajo hemos analizado algunos de los procesos educativos de la Educación Secundaria en Huelva. En concreto, nos hemos centrado en comprender aquellos que desembocan en fracaso escolar. Se ha observado la posible asociación entre el rendimiento educativo y los factores individuales, familiares y de organización escolar que ya fueron identificados por distintas aproximaciones teóricas anteriormente. El trabajo empírico se llevó a cabo en once Institutos de Educación Secundaria. Mediante el uso de una metodología mixta efectuamos un análisis micro de los factores centrados en el alumnado y un análisis meso de los factores referidos a los centros educativos. Los resultados evidenciaron la relevante dimensión del fracaso escolar en este contexto educativo. Se identificaron dos factores claves asociados de esta problemática educativa. En primer lugar, el grado de implicación familiar en el proceso educativo y, en segundo lugar, la organización escolar. Hemos observado que la prevención del fracaso escolar requiere de una actuación en dos líneas diferenciadas. Por una parte, la revisión de los criterios de agrupamiento escolar y designación tutorial que llevan a cabo los centros educativos. Y en segundo lugar, una intervención individualizada con el alumnado en riesgo que se inicie con los primeros síntomas de desapego y que se base en la cooperación continúa entre familias y equipos docentes, de manera, que trasmitan a los estudiantes estrategias de aprendizaje que refuercen su motivación individual.
\end{abstract}

Descriptores: Fracaso escolar, Educación secundaria, Implicación escolar, Rendimiento académico, Educación inclusiva.

In this work we analyze the educational processes of the Secondary Education in Huelva. Mainly we focused in understanding those that end in school failure. To understand the school failure, we have observed the association between the educational performance and the individual, family factors and school organization that were previously identified by different theoretical approximations. The empirical work was carried out in eleven Institutes of Secondary Education. By using a mixed methodology, we could carry out a micro analysis of the factors referred to the student body and a meso-analysis of the factors referred to the centers. The results demonstrated the important dimension of the school failure in this context of education. Two key factors were identified associated of these educational problems. First of all, the level of familiar implication in the educational process and, secondly, the school organization. We have noticed that the school failure prevention requires proceeding in two different ways. On one hand, the review of the criteria of school grouping and tutorial designation that carry out the educational centers. On the other hand, an individualized intervention with the student body in risk that begins with the first symptoms of indifference and that is based on the constant cooperation between families and educational teams, so that they transmit to the students learning strategies that reinforce the individual motivation.

Keywords: School failure, Secondary education, Student engagement, Academic performance, Inclusive education.

*Contacto: ccarmen.marquez@gmail.com

ISSN: 1696-4713

www.rinace.net/reice/

revistas.uam.es/reice
Recibido: 1 de noviembre 2012

$1^{\text {a }}$ Evaluación: 13 de septiembre de 2012

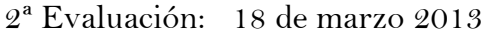

Aceptado: 1 de abril 2013 


\section{Introducción}

En muchos países de nuestro entorno se habla en la actualidad sobre equidad educativa. La equidad educativa puede definirse como "un estado del proceso educativo basado en condiciones, experiencias y situaciones pedagógicas orientadas a la obtención del éxito educativo de todo el alumnado" (Sánchez-Santamaría y Manzanares, 2014, p. 94). Hacer efectiva esta oportunidad de éxito educativo se ha convertido en uno de los objetivos prioritarios de las actuales políticas educativas. A pesar de ello, los resultados de las investigaciones recientes ponen de manifiesto los reiterados procesos de fracaso escolar que presentan nuestros estudiantes (Carabaña, 2012; De la Fuente y Jimeno, 2011; FEDEA, 2013; Navarrete, 2007; Sastre, 2015).

El fracaso escolar puede llegar a tener una especial repercusión en la vida de los estudiantes y en muchos casos fracaso escolar y social aparecen asociados. Hoy en día sabemos que fracasar en la escuela comporta un alto riesgo de ser excluido del contexto social normalizado (Azevedo, 2003).

Los procesos de fracaso escolar no emergen de forma repentina, suelen mostrase precedidos por conductas de desapego y de desvinculación progresiva que conducen al posterior abandono del sistema educativo (Fernández, Mena y Riviére, 2010; González, 2010). Este carácter previsible nos lleva a pensar que la correcta identificación de sus causas podría convertirse en el punto de partida desde el que adoptar medidas estructurales que fomenten la obtención del logro académico.

\section{Fundamentación teórica}

Elaborar una definición consensuada del fracaso escolar no ha sido posible hasta el momento. Esta problemática educativa se presenta como un concepto poliédrico que admite diversas interpretaciones en función del enfoque con el que se aborde. Algunos autores lo han definido en función de sus dimensiones, identificando una dimensión objetiva plasmada en los sistemas de evaluación y una dimensión subjetiva que explicaría por qué dos alumnos con una misma calificación podrían tener sentimientos de éxito o fracaso diferenciados (Perrenoud, 1990). Igualmente, al fracaso escolar se le ha atribuido componentes contextuales, determinados por las exigencias de acceso al mercado de trabajo, y componentes temporales, derivados de la evolución legislativa (Marchesi e Iglesias, 2008). Y se le ha presupuesto un valor denotativo y un valor connotativo por el que se estigmatiza al alumnado que fracasa (Fernández, Mena y Rivière, 2010).

La controversia generada en torno a la conceptualización del término ha provocado que algunos expertos se hayan mostrado contrarios a su utilización al considerar que ofrece una imagen negativa del alumnado, que daña su confianza y autoestima y que pone el énfasis en los resultados y no en la compresión de los procesos de aprendizaje (Gimeno y Carbonell, 2004).

La imprecisión conceptual que rodea al fracaso escolar se reproduce de manera similar en la identificación de los factores que lo facilitan o promueven. Esta cuestión ha generado un amplio debate entre expertos educativos y en la literatura de referencia podemos encontrar trabajos que destacan la influencia ejercida sobre el rendimiento educativo de factores de diversa naturaleza. Factores externos al alumnado como pueden ser un entorno socioeconómico desfavorable, métodos de enseñanza inadecuados o una 
actitud familiar desalentadora. Factores internos al estudiante que ponen el énfasis en las dificultades de atención y aprendizaje, el desinterés por la escuela o la dificultad de relación con iguales. $\mathrm{O}$ incluso la combinación de estos factores internos y externos ha sido identificada como causa del fracaso escolar (Moreno, 2009).

\subsection{El alumnado como sujeto activo del éxito escolar}

Si situamos al alumnado como sujeto activo del proceso educativo debemos hacer referencia a las múltiples aportaciones que han relacionado las características sociodemográficas de los estudiantes, tales como género, clase social o país de origen a su éxito o fracaso escolar (Hausmann, Tyson y Zahidi, 2009; Limones, 2011; MarcenaroGutiérrez y Navarro, 2011).Igualmente, aspectos más subjetivos como la motivación, las expectativas individuales o incluso los hábitos de estudio se ha relacionado con el rendimiento académico (Descals y Rivas, 2002; Navas, Sampascual y Santed, 2003). De estos últimos aspectos deriva un concepto que para nosotros es clave en el posterior rendimiento escolar, la implicación personal del alumnado en su proyecto educativo. Pensamos, tal y como recogen Simon-Morth y Chen (2009) que "los aspectos individuales se han vinculado al grado en que el alumnado está implicado, conectado y comprometido con la escuela, a la vez que motivado para aprender y rendir" (p. 4). Por tanto, esta implicación se convierte en un concepto central para explicar el éxito y fracaso escolar. Pero, ¿qué entendemos por implicación educativa del alumnado? Como respuesta a esta interrogante destacamos las dimensiones de la implicación educativa categorizadas por Frederiks, Blumfield y Paris (citadas en González, 2010) y que hacen referencia a la dimensión conductual, referida a las conductas observables que exhibe el alumnado y que indican su nivel de participación en la escuela. La dimensión afectiva, que alude a sus conexiones afectivas o emocionales que se desarrollan en la escuela. Y la dimensión cognitiva, entendida como la inversión psicológica y el esfuerzo que el estudiante dirige al aprendizaje, a la comprensión y al conocimiento.

\subsection{La familia promotora educativa}

De manera similar, la estructura familiar y el nivel educativo de los padres también se han relacionado frecuentemente con el rendimiento educativo del alumnado (Martínez y Álvarez, 2005; Martínez-Otero, 1997; Peralbo y Fernández, 2003). El capital social familiar fue asociado a la autoeficacia académica por Bourdieu y Passeron (1970) que detectaron una mayor incidencia del fracaso escolar entre el alumnado de la clase obrera. No solo las características estructurales de la familia han sido analizadas intentando comprender el fracaso escolar de los estudiantes, sino que también las relaciones intrafamiliares y la implicación de la propia familia en la escuela se identificaron como elementos influyentes en el discurrir educativo del alumnado. Algunos trabajos han asegurado que el clima familiar incide en el autoconcepto, en la autoestima, en la autoeficacia y en las conductas de autorregulación de los estudiantes incidiendo, consecuentemente, en el aprendizaje y rendimiento académico (Castejón y Pérez, 1998; Galicia, Sánchez y Robles, 2013; García, Pelegrina y Lendínez, 2002). Se ha considerado que la influencia de la implicación familiar en el proceso educativo es lo tan relevante que las expectativas familiares sobre el logro educativo pueden disminuir la probabilidad de abandono temprano (González-Pienda y Núñez, 2005; Muller, 1998). Uno de los trabajos más destacables en este sentido es el llevado a cabo por Martínez-Pons (1996). En este trabajo se analizó el significado de la implicación familiar en base al concepto de autoeficacia desarrollado por Zimmerman (1989) y se definió la implicación familiar en el 
proceso de autorregulación a través de cuatro tipos de conductas: las conductas de modelado (los padres ofrecen ejemplos que pueden ser imitados por los hijos/as), las conductas de estimulación (los padres favorecen la persistencia de los hijos ante condiciones adversas), las conductas de facilitación (los padres facilitan el aprendizaje aportando recursos y medios) y las conductas de recompensa (los padres recompensan las conductas que implican autorregulación).

\subsection{La organización escolar}

La organización escolar juega un papel decisivo en el desarrollo educativo de sus miembros. A través de ella puede concretarse la construcción de una escuela inclusiva y reductora de desigualdades (González, 2008; Lorente, 2006; Muñoz, Rodríguez y Barrera, 2013). Los modelos de dirección educativa, la formación del profesorado, los métodos de enseñanza-aprendizaje o las relaciones personales que se establecen en el interior de las aulas contribuyen a que los procesos educativos sean ricos y valiosos para todos (Baerveldt, Van de Bunt y de Federico, 2010; González, 2002; McMahon, 2009; Murillo y Hernández-Castilla, 2011). De esta forma, la escuela cumpliría su función social a través de un currículo y una organización escolar al servicio de la didáctica pero lamentablemente, esta no parece ser la realidad de un sistema educativo que, como el nuestro, no garantiza un contexto motivador de aprendizaje que minimice los riesgos de fracaso escolar (Birbili 2005; Calero, 2006).

En este trabajo entendemos el fracaso escolar como un fenómeno complejo, estructural y dinámico que se concreta en la incapacidad del alumnado para alcanzar los objetivos establecidos por el propio sistema educativo (Cernadas y Marsó, 2014; García, Casal y Planas, 1998; Martínez, 2007). De acuerdo con Escudero (2005), consideramos que el fracaso escolar no es un fenómeno natural sino una realidad construida en y por la escuela, en sus relaciones con los estudiantes y, naturalmente, de éstos con ella. En consecuencia, para ser capaces de comprenderlo debemos analizarlo desde una perspectiva holística que integre a todos los agentes implicados en el contexto escolar (González, 2009). Solo a través de esta aproximación integral podremos obtener resultados que nos lleven a elaborar nuevas prácticas educativas que propicien su erradicación.

El desarrollo de nuestra práctica profesional no posibilitó conocer múltiples escenarios educativos en los que observamos convergían tres circunstancias favorecedoras de los procesos de fracaso escolar. Encontramos a un alumnado que no se sentía integrado ni motivado por aprender, a docentes que hacían frente a la diversidad sin recursos ni formación para ello y a padres y madres que manifestaban serias limitaciones para resolver las situaciones de desajuste que se daban entre sus hijos y los centros escolares. Este panorama motivó nuestro deseo de acercarnos al contexto escolar de Educación Secundaria de Huelva y de profundizar en la naturaleza de los procesos de fracaso escolar.

Este trabajo se ha desarrollado con el objetivo de identificar la posible influencia de factores individuales, familiares y de organización escolar sobre el rendimiento académico del alumnado de Educación Secundaria en la provincia de Huelva. 


\section{Método}

La complejidad de la realidad abordada exigía la utilización de un enfoque multimetodológico (Ruiz, 2008). Para alcanzar nuestro objetivo de investigación aplicamos una metodología mixta que nos permitió obtener, analizar y vincular datos de carácter cualitativo y cuantitativo de forma complementaria.

\subsection{Muestra}

Una muestra representativa cuantitativa y cualitativamente será requisito imprescindible para la generalización de los resultados (Colás y Buendía, 1994). Por tanto, teniendo en cuenta que nuestro universo lo constituía el alumnado de Educación Secundaria de la provincia de Huelva, realizamos un muestreo estratificado polietápico con afijación proporcional para obtener nuestra muestra. Consideramos conglomerados los centros en función de dos indicadores: la tasa de promoción educativa por municipios facilitada por la Delegación Provincial de Educación de la Junta de Andalucía y el volumen de matriculación que ya había sido conectado con anterioridad a las relaciones que se establecen en el interior de los centros educativos (González, 2006).

En base a estos indicadores generamos una clasificación de centros con alto, medio y bajo volumen de matriculación establecidos en municipios con alta, media y baja tasa de promoción. Seleccionamos un centro de cada una de las subcategorías resultantes, intentado que estuvieran representadas todas las comarcas territoriales provinciales y como resultado, obtuvimos una muestra conformada por 11 centros educativos en los que entrevistamos a 206 estudiantes y 11 representantes de los equipos directivos de cada uno de los centros seleccionados. La elección del grupo educativo participante en el trabajo de campo quedó en manos de los propios centros escolares.

La edad media de la muestra se situó en 14,7 años. La mayor parte de los estudiantes cursaban estudios de $2^{\circ}$ y $3^{\circ}$ curso de ESO, sin diferencias destacables en función del género (52,9\% masculino y el $47,1 \%$ femenino) pero con diferencias destacables en relación al país de origen. La mayor parte del alumnado, el 88,9\%, era de origen español y un reducido porcentaje provenía de algún país extranjero. Concretamente, el 6,3\% procedían de Europa del Este (Rumania y Polonia), el 3,4\% de América Latina (Brasil, Colombia y Venezuela) y el restante 1,4\% del Norte de África (Marruecos).

\subsection{Procedimiento de análisis}

Tras un extenso análisis de la literatura especializada y de los informes educativos obtenidos de la base de datos Séneca (base de datos donde se sistematiza toda la información relativa a la gestión de los centros docentes, los servicios de apoyo a la educación, la inspección educativa, los programas y las actividades del sistema educativo andaluz) diseñamos dos instrumentos acordes a nuestro objetivo de investigación. Por una parte, una encuesta dirigida al alumnado que contenía cuestiones relativas a sus características socioeducativas, a sus expectativas escolares y a la implicación de sus familias en sus procesos educativos. Y, por otra parte, una entrevista semiestructurada dirigida al equipo directivo sobre los criterios que usaba el centro para decidir cuestiones relativas a la organización escolar de los centros. La información recogida se plasmó en una matriz de datos del programa estadístico SPSS. Para cada estudiante asociamos los datos obtenidos de su encuesta personal, la información procedente de la entrevista a su 
centro educativo y la información que sobre evaluación educativa recogía la base de datos Séneca.

Tomando como base esa matriz realizamos dos tipos de análisis que detallamos a continuación:

- Análisis estadístico descriptivo de:

$\checkmark$ Las características sociodemográficas

$\checkmark$ La motivación y expectativas educativas del alumnado

$\checkmark$ El nivel socioeducativo e implicación escolar familiar

$\checkmark$ Los criterios de organización escolar de sus respectivos centros educativos.

- Análisis bivariable (tablas de contingencia y prueba Chi-cuadrado) con el objetivo de comprobar la asociación entre los factores anteriormente citados y el rendimiento académico del alumnado. Para este segundo nivel de análisis tomamos como variable dependiente el rendimiento académico del alumnado y como variables independientes todas las posibles variables que la revisión bibliográfica había identificado como asociadas a este rendimiento. Estas variables se encuentran detalladas a continuación (tabla 1).

Tabla 1. Relación de variables independientes incluidas en el análisis bivariable

\begin{tabular}{|c|c|c|c|}
\hline \multicolumn{4}{|c|}{ FACTORES INDIVIDUALES DEL ALUMNADO } \\
\hline Sociodemográficos & \multicolumn{3}{|c|}{ Implicación y expectativas educativas } \\
\hline Género & Dimensión conceptual & Dimensión afectiva & Dimensión cognitiva \\
\hline Edad & Rto. académico & Clima escolar percibido & Utilidad de estudios \\
\hline Sexo & ión de curso & Valoración de los & Intención abandono \\
\hline País de procedencia & $\begin{array}{l}\text { Participacion } \\
\text { estructuras educ. }\end{array}$ & recursos del centro & Expectativas educ. \\
\hline \multicolumn{4}{|c|}{ FACTORES FAMILIARES } \\
\hline Modelado & Estimulación & Facilitación & Refuerzo \\
\hline $\begin{array}{c}\text { Nivel educativo } \\
\text { de los padres }\end{array}$ & $\begin{array}{l}\text { Interés por las relaciones y } \\
\text { tareas para casa } \\
\text { Interés resultados escolares }\end{array}$ & $\begin{array}{l}\text { Apoyo en la } \\
\text { elaboración de las } \\
\text { tareas para casa }\end{array}$ & $\begin{array}{l}\text { Recompensas y } \\
\text { castigos por los } \\
\text { resultados escolares }\end{array}$ \\
\hline \multicolumn{4}{|c|}{ FACTORES ORGANIZACIÓN DE CENTRO } \\
\hline \multicolumn{2}{|c|}{$\begin{array}{l}\text { Criterios de agrupación del alumnado } \\
\text { Criterios para la gestión de la acción tutorial } \\
\text { Criterios para la elección de grupo por el } \\
\text { profesorado }\end{array}$} & \multicolumn{2}{|c|}{$\begin{array}{l}\text { Criterios para la distribución del horario } \\
\text { escolar } \\
\text { Programas de educación compensatoria }\end{array}$} \\
\hline
\end{tabular}

Fuente: Elaboración propia.

\section{Resultados}

El primer resultado a destacar se relaciona con la importante dimensión del fracaso escolar en nuestro contexto de estudio. El porcentaje de fracaso escolar registrado entre nuestros estudiantes ascendió hasta el $43,9 \%$ del total.

A continuación, mostramos los resultados derivados del análisis bivariable realizado. En primer lugar, nos detenemos en las variables relativas a la dimensión individual, en concreto en los factores sociodemográficos como el sexo o país de origen que no mostraron asociación estadísticamente significativa con el rendimiento. En cuanto al 
sexo, ambos grupos registraron porcentajes similares de fracaso escolar, $23,8 \%$ alumnos y 20,1\% alumnas y tampoco se apreciaron diferencias significativas en cuanto al país de origen. El porcentaje de alumnado español con fracaso fue del $41 \%$ y el porcentaje de alumnado extranjero fue del $43,5 \%$ respectivamente.

En cambio, sí mostraron asociación estadísticamente significativa con el rendimiento académico las dos variables de la dimensión conductual analizadas (repetición de curso anterior y participación en las estructuras educativas). De esta forma, hallamos que el $45 \%$ del alumnado que presentó fracaso escolar manifestó haber repetido algún curso anteriormente. Y que el $80 \%$ del alumnado que participaba en las estructuras escolares (consejo escolar y delegación de alumnos) presentaba buen rendimiento académico mientras que solo el 20\% de ellos/as no promocionaba de curso.

En cuanto a la dimensión afectiva, la variable "aspectos más valorados del centro" también se mostró asociada significativamente al rendimiento académico. En este caso, comprobamos que el alumnado que promocionaba, al finalizar el curso, había citado como aspectos que más valoraba "la relación con el profesorado" y "la posibilidad de aprender" (en el $92 \%$ y $71,4 \%$ de los casos) mientras que la mayor parte del alumnado que no promocionaba había citado en mayor porcentaje "las actividades extraescolares" como el aspecto más valorado de su centro educativo (en el 73,3\% de los casos).

En relación a la dimensión cognitiva, las tres variables analizadas mostraron asociación estadísticamente significativa con el rendimiento académico. La utilidad atribuida por los estudiantes a los estudios apareció asociada a su rendimiento. El 73,8\% de quienes consideraban los estudios muy útiles para su vida futura promocionaron de curso mientras que el $90 \%$ de quienes los consideraban poco útiles presentaron fracaso escolar. En la misma línea, los resultados revelaron que la intención de abandono prematuro aparecía significativamente asociada al rendimiento del alumnado. Entre el alumnado que valoraba la posibilidad de abandonar la ESO sin concluirla, el 67,3\% presentaba fracaso escolar mientras que el $32,7 \%$ promocionaba. Igualmente, observamos que la aspiración educativa del $67,7 \%$ del alumnado que promocionaba se situaba en la obtención de un título universitario mientras que el 53,3\% que no promocionaba situaba su aspiración en la obtención del título de ESO.

En referencia al análisis relativo a los factores familiares, destacamos que el nivel educativo de los padres y madres no apareció asociado estadísticamente significativa al rendimiento del alumnado. Por el contrario, sí mostraron asociación las conductas de estimulación y refuerzo que los padres desarrollan. Entre las conductas de estimulación destaca el interés familiar por las relaciones en la escuela. El 59,6\% del alumnado cuyos padres se interesan "a menudo" por sus tareas para casa promocionaron de curso mientras que el $64,3 \%$ de quienes manifestaron que sus padres "nunca" se interesaban por ellas presentaron fracaso escolar. Igualmente, el $61,4 \%$ del alumnado con buen rendimiento académico manifestaba que sus padres se interesaban "a menudo" por sus resultados escolares y el 62,5\% del alumnado que fracasaba admitía que sus padres nos se interesaban "nunca" por su rendimiento escolar.

Las conductas de recompensa no mostraron asociación significativa con el rendimiento académico. Sin embargo, sí aparecieron asociadas las conductas de castigo. El 80\% del alumnado que manifestaba ser castigado si obtenía malos resultados académicos, promocionaba de curso. 
Por último, exponemos los resultados derivados del análisis entre el rendimiento académico y las características organizativas de los centros. Estos resultados mostraron que el 63,6\% del alumnado que presentaba fracaso escolar cursaba estudios en centros que realizaban agrupamiento en función de su rendimiento académico de su alumnado. En cambio, el $71,4 \%$ de quienes promocionaron estaban matriculados en centros que agrupaban por orden alfabético. Por otra parte, más de la mitad del alumnado que promocionó, el 64,3\%, se concentraba en los centros que seguían la normativa vigente para la gestión de tutorías mientras que los centros que seguían otros criterios registraron una menor tasa de promoción, situada en el 35,7\% de su alumnado. La mayor proporción de fracaso escolar, el $64,8 \%$, se concentró en los centros que utilizaban la antigüedad del profesorado como criterio para la elección. Y la mayor tasa de promoción educativa se registró en los centros cuyo profesorado elegía grupo escolar en función del criterio de dirección o departamento, el $66,7 \%$ y $45 \%$ respectivamente. Todos estos resultados se detallan a continuación (tabla 2 ).

Tabla 2. Resultados análisis bivariable sobre el rendimiento académico del alumnado

\begin{tabular}{|c|c|c|c|c|}
\hline & & $\mathbf{X}^{2}$ & $\begin{array}{c}\text { PROMOCIONA } \\
\%\end{array}$ & $\begin{array}{c}\text { REPITE } \\
\%\end{array}$ \\
\hline \multicolumn{5}{|l|}{ Sociodemográficos } \\
\hline Sexo alumnado & $\begin{array}{l}\text { Hombre } \\
\text { Mujer }\end{array}$ & $0,276^{\wedge}$ & $\begin{array}{l}55,6 \\
59,2\end{array}$ & $\begin{array}{l}44,4 \\
40,8\end{array}$ \\
\hline País de origen alumnado & $\begin{array}{l}\text { Nacionales } \\
\text { Extranjeros }\end{array}$ & $2,016^{\wedge}$ & $\begin{array}{l}59,0 \\
56,5\end{array}$ & $\begin{array}{l}41,0 \\
43,5\end{array}$ \\
\hline \multicolumn{5}{|l|}{ Dimensión Conductual } \\
\hline $\begin{array}{l}\text { Repetición de curso } \\
\text { anterior }\end{array}$ & $\begin{array}{l}\mathrm{Si} \\
\mathrm{No}\end{array}$ & $3,602^{*}$ & $\begin{array}{l}39,0 \\
63,2\end{array}$ & $\begin{array}{l}61,0 \\
36,8\end{array}$ \\
\hline $\begin{array}{l}\text { Participación activa en } \\
\text { escuela }\end{array}$ & $\begin{array}{l}\text { Sí } \\
\text { No }\end{array}$ & $0,147 *$ & $\begin{array}{l}80,0 \\
56,3\end{array}$ & $\begin{array}{l}20,0 \\
43,7\end{array}$ \\
\hline \multicolumn{5}{|l|}{ Dimensión Afectiva } \\
\hline \multirow{4}{*}{ Valoración de vida escolar } & \multirow{4}{*}{$\begin{array}{l}\text { Posibilidad aprender } \\
\text { No posib. aprender } \\
\text { Si activ. extraescol. } \\
\text { No activ. extraescol. } \\
\text { Sí relación con prof. } \\
\text { No relación con prof. } \\
\text { Sí relación comp. } \\
\text { No relación comp. }\end{array}$} & $13,332 * * *$ & $\begin{array}{l}71,4 \\
46,1\end{array}$ & $\begin{array}{l}28,6 \\
53,9\end{array}$ \\
\hline & & $6,031^{*}$ & $\begin{array}{l}26,7 \\
59,3\end{array}$ & $\begin{array}{l}73,3 \\
40,7\end{array}$ \\
\hline & & $5,404^{*}$ & $\begin{array}{l}92,0 \\
55,8\end{array}$ & $\begin{array}{r}8,0 \\
44,2\end{array}$ \\
\hline & & $0,437^{\wedge}$ & $\begin{array}{l}60,0 \\
55,4\end{array}$ & $\begin{array}{l}40,0 \\
44,6\end{array}$ \\
\hline \multicolumn{5}{|l|}{ Dimensión Cognitiva } \\
\hline $\begin{array}{l}\text { Utilidad de los estudios } \\
\text { para la vida futura }\end{array}$ & $\begin{array}{l}\text { Muy útiles } \\
\text { Útiles } \\
\text { Poco útiles }\end{array}$ & $50,984^{* * * *}$ & $\begin{array}{l}73,8 \\
52,5 \\
10,0\end{array}$ & $\begin{array}{l}26,2 \\
47,5 \\
90,0\end{array}$ \\
\hline $\begin{array}{l}\text { Posibilidades de abandono } \\
\text { de los estudios } \\
\text { obligatorios }\end{array}$ & $\begin{array}{l}\text { Si es posible } \\
\text { No es posible }\end{array}$ & $15,936^{* * *}$ & $\begin{array}{l}32,7 \\
65,0\end{array}$ & $\begin{array}{l}67,3 \\
35,0\end{array}$ \\
\hline $\begin{array}{l}\text { Expectativas educativas de } \\
\text { futuro }\end{array}$ & $\begin{array}{l}\text { ESO } \\
\text { Bachillerato } \\
\text { E. Universitarios } \\
\text { Ns/Nc }\end{array}$ & $7,840^{*}$ & $\begin{array}{l}46,7 \\
50,0 \\
67,7 \\
60,0\end{array}$ & $\begin{array}{l}53,3 \\
50,0 \\
32,3 \\
40,0\end{array}$ \\
\hline
\end{tabular}

Nota: $(\mathrm{n}=206)$ y $(\mathrm{n}=11) * \mathrm{p}<0.05,{ }^{*} * \mathrm{p}<0.01,{ }^{*} * * \mathrm{p}<0.001,{ }^{\wedge}:$ no significativo.

Fuente: Elaboración propia. 
Tabla 2. Resultados análisis bivariable sobre el rendimiento académico del alumnado. Continuación

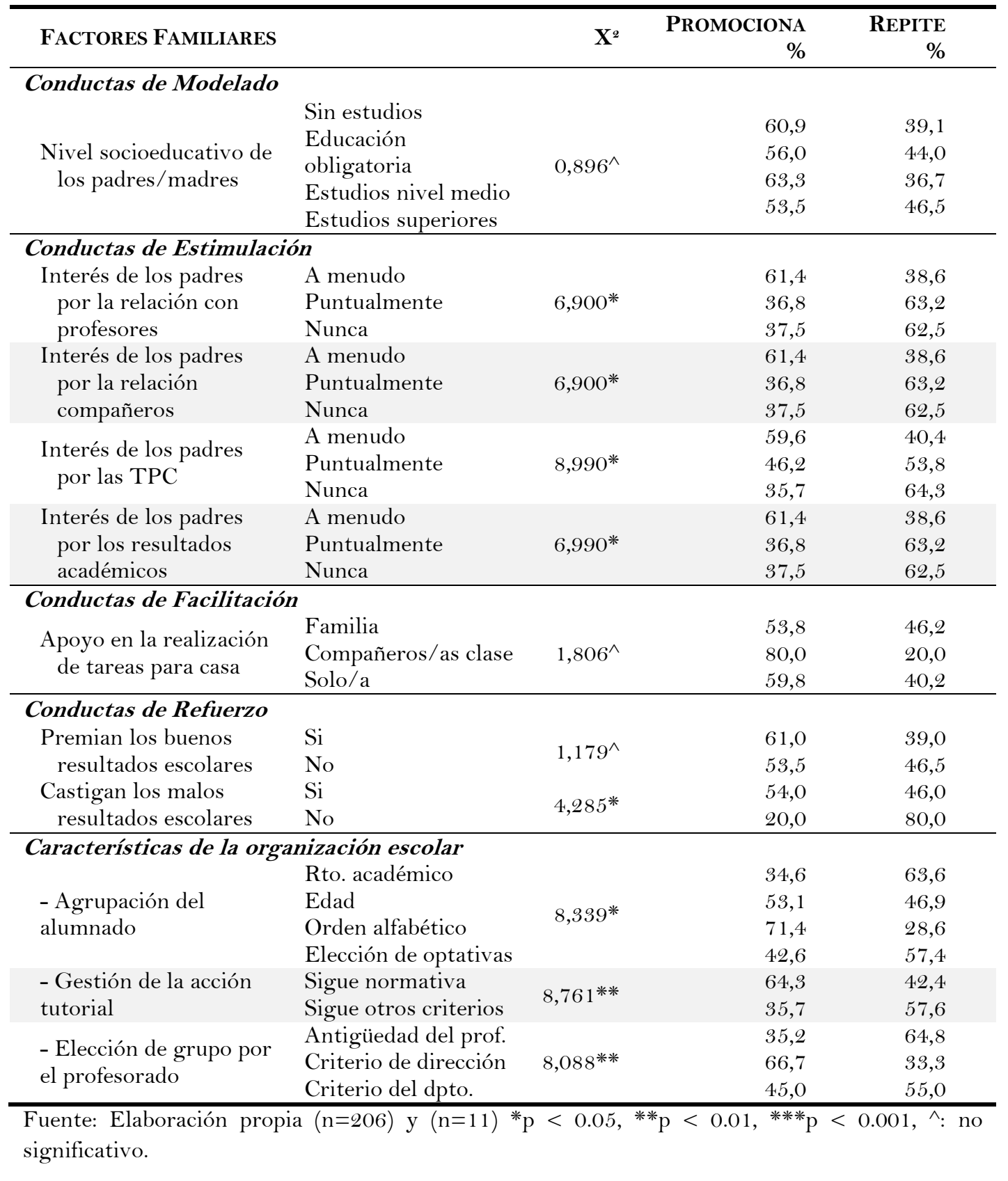

En base a los resultados descritos hemos podido diferenciar dos grupos de estudiantes con características claramente diferenciadas y que pasamos a describir. Entre el alumnado encuestado, identificamos un primer grupo de estudiantes que manifestaron tener una alta consideración de la utilidad de los estudios para el desarrollo de sus proyectos futuros. Estos estudiantes no valoraban la posibilidad de abandonar los estudios sin concluirlos y su aspiración educativa se situaba en la obtención de estudios universitarios. A nivel académico, comprobamos que la mayor parte de este grupo presentó buen rendimiento académico y promocionó de curso al finalizar el año escolar. En contraposición, identificamos un segundo grupo compuesto, en su mayor parte, por estudiantes que manifestaron una menor consideración a la utilidad de los estudios en 
sus vidas y cuya aspiración educativa se situaba en la obtención del título de Bachillerato y/o Formación Profesional. Estos alumnos/as admitieron valorar la posibilidad de abandonar prematuramente su escolarización. A nivel académico pudimos comprobar que gran parte de ellos/as presentaron un bajo rendimiento académico que les obligó a repetir curso al finalizar el año escolar. Hemos representado estos datos gráficamente a continuación (gráfico 1).

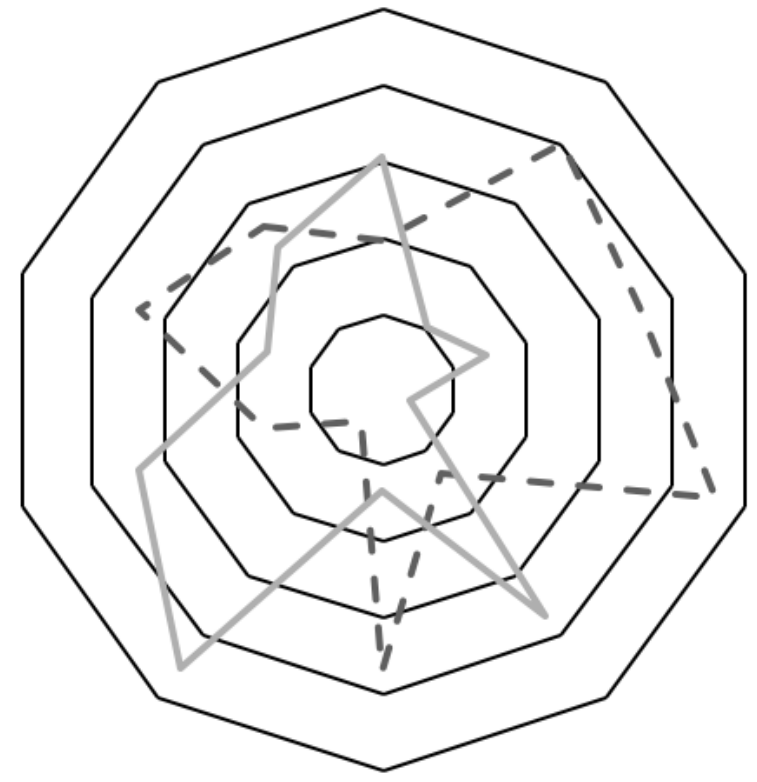

- Alumando promociona Alumnado no promociona

Gráfico 1. Perfiles educativos del alumnado en función del rendimiento académico Fuente: Elaboración propia $(\mathrm{n}=206)$.

\section{Conclusiones}

Aun sabiendo que nuestros resultados no pueden generalizarse al tratarse de un estudio de caso, queremos reflexionar sobre lo observado en nuestra realidad educativa. En primer lugar, queremos destacar la importante dimensión del fracaso escolar en nuestra provincia que lo convierte en una de las grandes problemáticas a la que nos enfrentamos. Esta destacable presencia de los procesos de fracaso escolar entre nuestros estudiantes hace imposible que podamos hablar de equidad en nuestro entorno educativo. En segundo lugar, los resultados evidencian la necesidad de una estrategia de actuación conjunta que cuente con el compromiso y la implicación de todos los agentes educativos como estrategia preventiva ante el fracaso escolar.

El fracaso escolar lejos de aparecer como un fenómeno que se reproduce de forma idéntica en cualquier entorno educativo parece estar muy definido por las particulares características contextuales en las que se desarrolla. Por esta razón, para su abordaje resulta imprescindible realizar un trabajo primer trabajo de identificación de los factores que facilitan su posterior desarrollo. Este análisis preliminar nos ayudará a desarrollar una estrategia de prevención coherente y adaptada que minimice sus efectos sobre el rendimiento académico del alumnado. Paralelamente debe iniciarse el trabajo individualizado con el alumnado en riesgo en cuanto se manifiesten los primeros síntomas de desapego (Calero, Choi y Waisgrais, 2010; Tinajas, 2009). No debemos 
esperar a que se desencadene un proceso de repeticiones sucesivas que no mejorará su rendimiento y que sumergirá al estudiante en una espiral de fracaso que concluirá en el posterior abandono escolar (OCDE, 2010).

A nivel de organización de centro se hace evidente la necesidad de una nueva configuración de la escuela centrada solo y exclusivamente en el alumno/a. Una nueva configuración centrada en identificar las barreras que dificultan la participación del alumnado en condiciones de igualdad, prestando una especial atención al alumnado en riesgo de exclusión (Booth et Ainscow, 2002). Para ello, la administración educativa debe dotar a los centros de Educación Secundaria de recursos que posibiliten una atención individualiza del alumnado y la puesta en marcha procesos de innovación que promuevan su participación en los centros (Echeita, 2009).

Igualmente, se hace imprescindible que los centros reflexionen sobre prácticas tan discriminantes como la agrupación en función del rendimiento o la asignación de profesor a grupo educativo en función de su antigüedad. Estas prácticas responden a intereses ajenos al alumnado y favorecen el desarrollo sistemático de los procesos de fracaso escolar (Cucurella, 2002; González, 2002; Feito, 2000).

En este trabajo constatamos que el aprendizaje está estrechamente relacionado con la motivación y las expectativas de los propios estudiantes (Schunk y Zimmerman, 2008) y que ambos elementos se ven reforzados cuando los estudiantes perciben interés y atención por parte de sus familias y de sus docentes. Se requiere el trabajo conjunto de los centros educativos y las familias en una relación de cooperación que trasmita a los estudiantes estrategias de aprendizaje y refuerce su motivación personal.

\section{Referencias}

Azevedo, J. (2003). Entre la escuela y el mercado de trabajo. Una mirada crítica sobre las transiciones. En A. Marchesi y C. Hernández (Coords.), El fracaso escolar. Una perspectiva internacional (pp. 203-245). Madrid: Alianza.

Baerveldt, C., Van de Bunt, G. y de Federico, A. (2010). Why and how selection patterns in classroom networks differ between students. Redes: Revista Hispana para el Análisis de Redes Sociales, 19, 272-300.

Birbili, M. (2005). Constants and contexts in pupil experience of schooling in England, France and Denmark. European Educational Research Journal, 4(3), 313-320. doi:10.1080/03050060126690

Booth, T. y Ainscow, M. (2002). Guía para la evaluación y mejora de la evaluación inclusiva. Madrid: Consorcio Universitario para la Educación Inclusiva.

Bourdieu, P. y Passeron, J. (1970). La reproducción. Elementos para una teoría del sistema de enseñanza. Barcelona: Ediciones Laia.

Calero, J. (2006). Desigualdades tras la educación obligatoria: nuevas evidencias. Documento de Trabajo de la Fundación Alternativas, 83(6), 56-78.

Calero, J., Choi, A. y Waisgrais, S. (2010). Determinantes del fracaso escolar en España: una aproximación a través de un análisis multinivel aplicado a PISA 2006. Revista de Educación, número extraordinario, 225-256. 
Carabaña, J. (2012). Debilidades de PISA y errores en la atribución del fracaso escolar académico. En M. de Puelles Benítez (Coord.), El fracaso escolar en el estado de las autonomías (pp. 155181). Madrid: Wolters Kluwer.

Castejón J. L. y Pérez A. M. (1998). Un modelo causal-explicativo de las variables psicosociales en el rendimiento académico. Revista Bordón, 5O(2), 174-185.

Cernadas, A. y Marsó, M. (2014). Un análisis del fracaso escolar en dos centros de Educación Secundaria. Revista de Estudios e Investigaciones en Psicología y Educación, 1(2), 122-131. doi:10.17979/reipe.2014.1.2.8

Colás, M. y Buendía, L. (1994). Investigación educativa. Sevilla: Ediciones Alfar.

Cucurella, S. (2002). La diversitat com a avantatge. La secundària a examen. Barcelona: Edicions Proa.

De la Fuente A. y Jimeno J. F. (2011). La rentabilidad privada y fiscal de la educación en España y sus regions. En A. Cabrales y A. Ciccone (Coords.), La educación en España. Una visión académica (pp. 230-271). Madrid: Monografías FEDEA

Descals, A. y Rivas, F. (2002). Capacidades intelectuales y rendimiento escolar en secundaria: constatación de una limitada relación. Revista Galego-Portuguesa de Psicoloxia e Educacion, $6(8), 203-214$.

Echeita, G. (2009). Los procesos de inclusión educativa desde la Declaración de Salamanca. Un debate entre doloroso y esperanzado. En C. Giné (Coord.), La educación inclusiva. De la exclusión a la plena participación de todo el alumnado (pp. 166-187). Barcelona: Horsori Editorial.

Escudero, J. M. (2005). El fracaso escolar: nuevas formas de exclusión educativa, ¿de qué se excluye y cómo? Profesorado. Revista de Curriculum y Formación del Profesorado, 1(1), 1-24

FEDEA. (2013). El informe PIAAC. Boletín observatorio educación. Recuperado de http://www.fedea.net/educacion/OEF/OEF 1.pdf

Feito, R. (2000). Los retos de la escolaridad obligatoria. Madrid: Editorial Ariel.

Fernández, M., Mena, L. y Riviere, J. (2010). Fracaso y abandono escolar en España. Barcelona: Fundación La Caixa.

Galicia, I., Sánchez, A. y Robles, F. (2013). Autoeficacia en escolares adolescentes: su relación con la depresión, el rendimiento académico y las relaciones familiares. Anales de Psicología, 29(2), 491-500. doi:10.6018/analesps.29.2.124691

García, M., Casal, J. y Planas, J. (1998). Las reformas en los dispositivos de formación para combatir el fracaso escolar en Europa. Revista de Educación, 317, 301-317.

García, M. C., Pelegrina, S. y Lendínez, J. (2002). Los estilos educativos de los padres y la competencia psicosocial de los adolescentes. Anuario de Psicología, 33, 79-95. doi: $10.1174 / 021037002317417796$

Gimeno, J. y Carbonell, J. (2004). El sistema educativo: una mirada crítica. Barcelona: Praxis.

González, M. T. (2002). Agrupamiento de alumnos e itinerarios escolares: cuando las apariencias engañan. Educar, 29, 167-182.

González, M. T. (2006). Absentismo y abandono escolar: una situación singular de la exclusión educativa. REICE. Revista Iberoamericana sobre Calidad, Eficacia y Cambio en Educación, 4(1), 1-15. 
González, M. T. (2008). Diversidad e inclusión educativa: algunas reflexiones sobre el liderazgo en el centro escolar. REICE. Revista Iberoamericana sobre Calidad, Eficacia y Cambio en Educación, 6(2) 82-99.

González, M. T. (2009). El abandono escolar: etiología, causas y vías para su reducción. Escuela Española, 3, 7-8.

González, M. T. (2010). El alumno ante la escuela y su propio aprendizaje: algunas líneas de investigación en torno a la implicación. REICE. Revista Iberoamericana sobre Calidad, Eficacia y Cambio en Educación, 8(4), 10-31.

González-Pienda, J. A. y Núñez, J. C. (2005). La implicación de los padres y su incidencia en el rendimiento de los hijos. Revista de Psicología y Educación, 1(1), 115-134.

Hausmann, R., Tyson, L. y Zahidi, S. (2009). The global gender gap report 2009. Ginebra: World Economic Forum.

Limones, I. (2011). Adolescentes y percepción del sí mismo: la construcción de una imagen realista de la adolescencia desde la familia la escuela.Etic@net, 11,233-252.

Lorente, A. (2006). Cultura docente y organización escolar en los institutos de secundaria. Profesorado. Revista de Currículum y Formación del Profesorado, 10(2), 1-13.

Marcenaro-Gutiérrez, O. y Navarro, C. (2011). El rendimiento educativo del alumnado en secundaria: ¿cuestión de género? Cuadernos de Ciencias Económicas y Empresariales, 61, 3970.

Marchesi, A. e Iglesias, E. (2008). Metas educativas 2021: la educación que queremos para la generación de los bicentenarios. Transatlántica de Educación, 5, 11-129.

Martínez, J. (2007). Clase social, género y desigualdad de oportunidades educativas. Revista de Educación, 342, 287-306.

Martínez, R. y Álvarez, L. (2005). Fracaso y abandono escolar en la Educación Secundaria Obligatoria: implicación de las familias y centros escolares. Aula abierta, 85, 127-146.

Martínez-Otero, V. (1997). Los adolescentes ante el estudio. Causas y consecuencias del rendimiento académico. Madrid: Editorial Fundamentos.

Martínez-Otero, V. (2009). Diversos condicionantes del fracaso escolar en la Educación Secundaria Obligatoria. Revista Iberoamericana de Educación, 51, 67-85.

Martinez-Pons, M. (1996). Test of a model of parental inducement of academic self-regulation. Journal of Experimental Education, 64, 213-227. doi:10.1080/00220973.1996.9943804

McMahon, W. (2009). Higher learning, great good: the private and public benefices of higher education. Baltimore (MD): John Hopkins University Press.

Moreno, M. (2009). ¿Por qué aprenden los estudiantes? Los objetivos de logro y su relación con el éxito o fracaso escolar. Zona Próxima, 11, 186-195.

Muller, C. (1998). Gender differences in parental involvement and adolescents' mathematics achievement. Sociology of Education, 71, 336-356. doi:10.2307/2673174

Muñoz, J., Rodríguez, D. y Barrera, A. (2013). Herramientas para la mejora de las organizaciones educativas y su relación con el entorno. Perspectiva Educacional, 52(1), 97-123.

Murillo, F. J. y Hernández-Castilla, R. (2011). Trabajar por la justicia social desde la educación. REICE. Revista Iberoamericana sobre Calidad, Eficacia y Cambio en Educación, 9(4), 4-16.

Navarrete, L. (2007). Jóvenes y fracaso escolar. Madrid: Instituto de la Juventud. 
Navas, L., Sampascual, G. y Santed, G. (2003). Predicción de las calificaciones de los estudiantes: la capacidad explicativa de la inteligencia general y de la motivación. Revista de Psicología General y Aplicada, 56, 225-237.

OCDE. (2010). PISA 2009 Results. París: OCDE.

Peralbo, M. y Fernández, L. (2003). Estructura familiar y rendimiento escolar en la educación secundaria obligatoria. Revista Galego-Portuguesa de Psicoloxia e Educación, 7(8), 309-323.

Perrenoud, P. (1990). La construcción del éxito y del fracaso escolar. Madrid: Morata.

Ruiz, C. (2008). El enfoque multimetodológico en la investigación social y educativa: una mirada desde el paradigma de la complejidad. Revista de Filosofía y Sociopolítica de la Educación, 8, 13-28.

Sánchez-Santamaría, J. y Manzanares, A. (2014). Tendencias internacionales sobre equidad educativa desde la perspectiva del cambio educativo. Revista de Investigación Educativa, $16(1), 12-28$.

Sastre, A. (2015). Iluminando el futuro: invertir en educación es luchar contra la pobreza infantil. Recuperado de: http://www.savethechildren.es/

Simons-Morton, B. y Chen, R. (2009). Peer and parent influences on school engagement among early adolescents. Touth E̊ Society, 41(1), 3-25. doi:10.1177/0044118x09334861

Schunk, D. y Zimmerman, B. (2008). Motivation and self-regulated learning: theory, research and applications. Nueva York: Lawrence Erlbaum.

Tinajas, A. (2009). La formación de grupos y la atención a la diversidad en un centro de enseñanza secundaria. Estudio de casos. Revista Iberoamericana de Educación, 50(3), 1-9.

Zimmerman, B. (1989). A social cognitive view of self-regulated academic learning. Journal of Educational Psychology, 81(3), 67-98. doi:10.1037/0022-0663.81.3.329

\section{Breve CV de la autora}

\section{Carmen Márquez Vázquez}

Doctora Europea en Ciencias Sociales por la Universidad de Huelva, obtuvo el título de doctora con la tesis "Fracaso escolar en la Educación Secundaria de Huelva" en 2014.

Desde 2009 se dedica al mundo de la docencia e investigación universitaria. Ha trabajado como profesora asociada en los departamentos de sociología y trabajo social de las Universidades de Huelva y Pablo Olavide de Sevilla durante más de cinco años y actualmente, en el departamento de Psicología Evolutiva y Educación de la Universidad Autónoma de Madrid y en el Máster de Psicopedagogía y Máster de Educación Secundaria en la Universidad Internacional de la Rioja. Interesada en la investigación educativa trabaja sobre la situación de los grupos vulnerables (niñas, población inmigrante, absentistas) dentro del sistema escolar. Durante más de quince años ha trabajado como técnica de proyectos sociales y educativos en distintas administraciones públicas, desde el Instituto Andaluz de la Mujer a la Diputación de Huelva o la Delegación de Educación de la Junta de Andalucía. Email: ccarmen.marquez@gmail.com 